\title{
DIPHTHERIC HEART-DISORDERS IN CHILDREN
}

\author{
BY
}

\section{NEUBAUER, M.D.}

R.M.O., City Hospital for Infectious Diseases, Walker Gate, Newcastle-upon-Tyne

Among all the ill effects which diphtheria may cause in childhood the damage inflicted on the heart is the most serious. The vast majority of deaths in children due to this disease occur as a result of injury to the cardiovascular system. A few cases of the laryngeal type result in death from mechanical obstruction and some others from pharyngeal paralysis. Practically all the remaining deaths are due to some form of cardiac failure.

Knowledge of the pathology, clinical signs, and treatment of diseases of the heart has been much increased in recent years by electrocardiography, and it is proposed to give an account of 100 cases of diphtheria with myocardial lesions observed from the onset to either convalescence or termination. These fall into three groups: (1) lesions of the heart musculature ; (2) abnormalities of rhythm ; (3) interference with conductivity.

\section{The'Myocardial Lesion}

The clinical signs of this condition are well known. Chief among them are listlessness, pallor, and increase or decrease in the pulse rate. Albuminuria may be found, and when present to a marked extent during the early stages of the disease is regarded as a bad prognostic sign.

The Electrocardiogram.-Alterations of the T-wave, S-T stretch, or of both, are often found. The T-wave is low in comparison with the preceding P-summit, the height of which is normally half that of the $T$. In other cases the $T$-wave is either absent or inverted or diphasic, in which latter case the inverted part is followed by a final wave. These signs are regarded as pathological in children if they occur in lead I

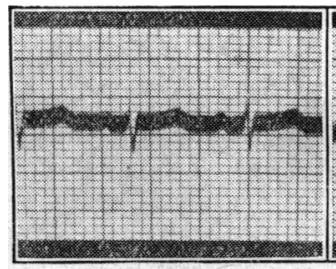

FIG. 1

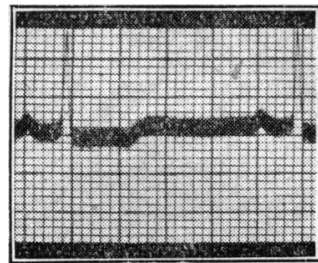

FIG. 3

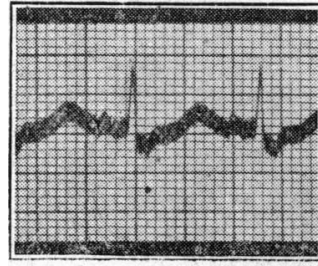

FIG. 5

Fig. 1.-T-wave very low

FIG. 2.-T almost isoelectric

Fig. 3.-S-T stretch dislocated, $T$ diphasic

Fig. 4.-S-T dislocated and almost horizontal.

FIG. 5-S-T a steep line, connecting $S$ with $T$

FIG. 6.-S-T dislocated, $T$ inverted.

or leads $I$ and $I I$, or in all three leads. It has been shown in recent years that the degree of dislocation of the S-T stretch is of as much importance as variations of the $T$-wave in myocardial lesions, perhaps of even greater importance. The
S-T stretch is compared with the level of the preceding $P-R$ stretch to estimate its dislocation. Slight dislocation of less than $0.5 \mathrm{~mm}$. may be disregarded, but any dislocation exceeding $1 \mathrm{~mm}$. is pathological. It must be remembered that variations of this type are seen normally in lead III, that a flat or diphasic $\mathrm{T}$-deflection may appear in lead II in children with meteorism, and that after treatment with digitalis the S-T stretch is dislocated and the T-wave flattened in lead II. If these variations are kept in mind the abnormalities in the electrocardiogram can be considered as due to myocardial damage caused by the diphtheric toxin. Indications of the pathological changes in the myocardium are sometimes found when the clinical signs are still doubtful. At the same time evidence of myocardial damage is sometimes still found when clinically the child appears to be quite recovered. The importance of the latter fact is obvious. At this hospital no child who has had a myocardial lesion is discharged until the electrocardiogram has returned to normal. Failure to ascertain that a myocardial lesion has completely cleared up may account for reports about slow recovery after diphtheria in certain cases, and for those tragic instances when a child dies suddenly at home after discharge from hospital. The electrocardiogram returns to normal again after 3 to 4 weeks in light cases, after 6 to 7 weeks in moderately severe cases, and after 10 to 13 weeks in severe cases in which a myocardial lesion is present without any disturbance of conductivity. In the present series of cases $38 \%$ show evidence of myocardial damage without any disturbance of conductivity. Some examples of variation in the $\mathrm{T}$-wave and $\mathrm{S}-\mathrm{T}$ stretch in cases of diphtheric myocarditis are shown in Figs. 1-6. All of them are lead II.

\section{Abnormalities of Rhythm}

Sinus Tachycardia.-Sinus tachycardia is very often seen during the febrile period of diphtheria, and is of no special significance. Tachycardia which persists into later convalescence should be carefully investigated. If the tachycardia is due to a myocardiăl lesion, evidence of the latter will be found together with the signs of the tachycardia.

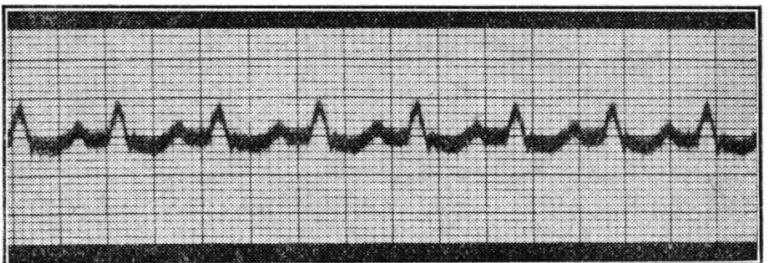

FIG. 7

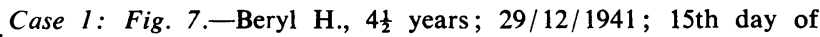
disease.

Lead II: Regular iachycardia, rate 120 . $\mathrm{R}$ notched; voltage $4 / 10 \mathrm{mV}$; duration $0.12 \mathrm{sec}$. $S-T$ below the base-line. $T$ diphasic.

Sinus Bradycardia.-Brac'ycardia is often found in the early convalescent stages of diphtheria as in other infectious diseases. The condition may, however, be due to cardiac damage. Gross bradycardia should always be carefully investigated to exclude a cardiac lesion. An electrocardiogram enables a differential diagnosis to be made at once.

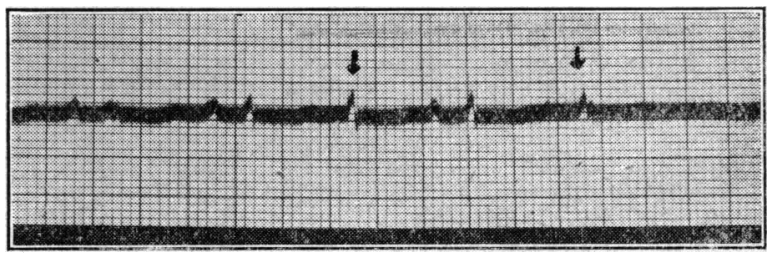

Fig. 8

Sinus Arrhythinia.-Sinus arrhythmia is a common cause of cardiac irregularity in childhood and has no pathological significance. It should be carefully distinguished from more serious forms of irregularity, as otherwise the activity of $a^{*}$ healthy child may be quite unnecessarily restricted.

Extrasystoles.-Extrasystoles are not generally regarded as of serious import, and to a certain point the same may be said of them when they occur during the course of diphtheria. 
Case 2: Fig. 8.-Robert L., 5 years; 22/8/1941; 22nd day of disease.

Lead II : P-R 0.23 sec. (partial a.-v. block). Ventricular complex of very low voltage; splintered and notched. $T$ inverted. There are two extrasystoles, indicated by arrows.

\section{Interference with Conductivity}

\section{The Auriculo-ventricular Block}

Partial a.-v. Block.-This is a serious condition in which there is a lesion either of the a.-v. node itself or of the bundle of His and the surrounding myocardium. It occurs in severe infections, mostly of the gravis type. Clinical evidence of the condition is limited to bradycardia together with enlargement of the heart, but a definite diagnosis is impossible without an electrocardiogram. This latter shows a lengthening of the $P-R$ interval, which normally in children does not exceed 0.12 second. A $\mathrm{P}-\mathrm{R}$ interval of 0.2 second or more must be regarded as definitely pathological. Partial a.-v. block was found in $17 \%$ of the present series.

Case 3: Fig. 9.-Rosalie B., 3 years; 18/10/1941; 13th day of disease.

Lead II : Action regular. P-P 1.47 sec. Rate 40 . P-R 0.36 sec. $R$ notched near the peak. S-T below the base-line. $T$ upright.

Complete a. $-v$. Block.-Clinical signs. An early symptom of the condition is often a syncopal attack in which the child is blanched and collapsed. The most marked feature is the extreme bradycardia, the pulse rate dropping to 30-40 a minute. Following a syncopal attack the patient remains pale and cyanotic, with coldness of the face and extremities and a livid tinge in the cheeks. The expression is anxious and there is often precordial or abdominal pain, together with marked restlessness and vomiting., Within one or two days the liver shows evidence of enlargement. Complete a.-v. block was found in $5 \%$ of the present series.

The electrocardiogram shows that the P-summits occur at equal intervals. The ventricular complexes also occur at equal intervals, but their rate is much less than that of the auricles. The varying length of the $P-R$ intervals shows that there is no connexion between the auricular and ventricular contractions.

Case 4: Fig. 10.-Rosalie B., 3 years; 20/10/1941; 15th day of disease.

Lead II : P-P $0.65 \mathrm{sec}$. Auricular rate 92. R-R $2.00 \mathrm{sec}$. Ventricular rate 30 . The auricle is beating three times more quickly than the ventricle. It is a 1:3 block. $R$ widened and notched; duration $0.18 \mathrm{sec}$; voltage $1 / 4 \mathrm{mV}$. T upright.

Lead III : P-P $0.65 \mathrm{sec}$. Auricular rate 92 . R-R $2.30 \mathrm{sec}$ Ventricular rate 26 . Duration of ventricular complex $0.14 \mathrm{sec}$. $T$ inverted.

\section{Bundle-branch Block}

Partial Bundle-branch Block.-In the present series $19 \%$ of the cases showed evidence of this condition, which, as in the case of partial auriculo-ventricular block, can only be definitely diagnosed by use of the electrocardiogram. This shows a prolonged duration of the ventricular deflection, which normally does not exceed 0.05 second. In this condition it may be prolonged to 1 second or more, while the ventricular. deflections are seen to be splintered and notched.

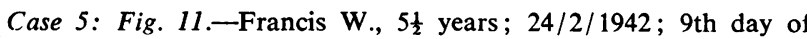
disease.

Lead I : Action regular. R-R 0.75 sec. P indiscernible. Ventricular complex of low voltage. $R$ splintered and thickened; duration

0.1 sec. $S-T$ below the base-line. $T$ isoelectric.

Lead II : P-P $0.80 \mathrm{sec}$. Rate 75 . P-R $0.20 \mathrm{sec}$. Ventricular complex splintered at the peak; voltage $6 / 10 \mathrm{mV}$; duration $0.1 \mathrm{sec}$ $\mathrm{S}-\mathrm{T}$ far below the base-line. $\mathrm{T}$ diphasic.

Lead III : R-R $0.80 \mathrm{sec}$. $\mathbf{P}$ indiscernible. Ventricular complex splintered and notched; voltage low; duration $0.05 \mathrm{sec}$. T isoelectric.

Complete Bundle-branch Block.-This condition occurred in $4 \%$ of the present series. The chief characteristics seen in the electrocardiogram are that $R, S$, and $T$ waves are of high voltage ; that a marked $R$-deflection is followed by an inverted $\mathrm{T}$, while an exaggerated S-deflection is followed by an upright $T$, and that the duration of the ventricular complex is prolonged together with splintering and notching of the deflections.

Case 6: Fig. 12.-Ronald R., 5 years; 4/9/1941; 10th day of disease.

Lead I: P-R 0.31 sec. $R$ splintered and notched; voltage 7/ $10 \mathrm{mV}$; duration 0.15 sec. $\mathrm{T}$ inverted.

Lead II : Voltage of $\mathrm{S} 1 \frac{1}{4} \mathrm{mV}$; duration $0.11 \mathrm{sec}$. T upright.
Lead III : Exaggerated S; voltage $1 \mathrm{mV}$; duration $0.14 \mathrm{sec} . \mathrm{T}$ upright.

Left bundle-branch block.

Case 7: Fig. 13.-Jean W., 2 5/12 years; 25/2/1942; 8th day of disease.

Lead I : P-P $0.82 \mathrm{sec}$. Auricular rate 73. S-S $2.39 \mathrm{sec}$. Ventricular rate 25 . S splintered; voltage $6 / 10 \mathrm{mV}$; duration 0.14 sec. T upright.

Lead II : Auricular deflection almost indiscernible. S-S $2.10 \mathrm{sec}$. Ventricular rate 28. Ventricular complex shows a notched downward deflection, the voltage of which is $1 / 3 \mathrm{mV}$, the duration $0.1 \mathrm{sec}$.

Lead III : Ventricular complex shows a very high $R$; voltage $1 \frac{1}{4} \mathrm{mV}$; duration $0.1 \mathrm{sec}$. $\mathrm{T}$ inverted.

Right bundle-branch block.

\section{Auricular Fibrillation}

The electrocardiogram has shown that auricular fibrillation does not only occur in severely damaged hearts, but may also be seen as a transitory phenomenon in children when the heart muscle is undamaged.

Transitory A uricular Fibrillation.-This condition may arise in children during diphtheria, and may last a few hours or a few days. No abnormal clinical signs can be detected either before or after the attack, and the patient is not disturbed or distressed. The prognosis is good. The electrocardiogram shows typical auricular fibrillation, but there are no other signs of myocardial damage. The condition occurred in $2 \%$ of the present series.

Case 8: Fig. 14.--Irene G., 5 years; $9 / 10 / 1941$; 29th day of disease.

Lead II : Action irregular. R-R 0.56 to $0.64 \mathrm{sec}$. Ventricular complex of normal configuration; voltage $8 / 10 \mathrm{mV}$; duration $0.05 \mathrm{sec}$. P-summits absent. The ventricular deflections are connected by oscillations. T-waves deformed by oscillations falling with them.

Lead III : Action irregular. R-R 0.60 to $0.63 \mathrm{sec}$. $P$ absent. Ventricular complex of normal configuration; voltage $6 / 10 \mathrm{mV}$; duration 0.05 sec.; connected by oscillations.

Two days later the electrocardiogram did not show anything abnormal. The child had a normal convalescence.

A uricular Fibrillation in Severe Heart Lesions.-Patients who develop this condition show signs of myocardial damage for a period, sometimes long and sometimes short, before the onset of auricular fibrillation. The prognosis is very poor, especially in those cases in which congestion was present before the fibrillation started. According to Lewis, "the auricular fibrillation loads the already defective muscle with an extra burden." The electrocardiogram shows all the signs of a severely damaged heart muscle.

Case 9: Fig. 15.-Robert B., 3 years; 12/2/1942; 55th day of disease.

Lead I: Action irregular. $P$ indiscernible. There are oscillations in the first cycle. Ventricular complex: voltage very low $(1 / 4 \mathrm{mV})$. $S-T$ on the base-line. $T$ round.

Lead II: Action irregular. $\mathbf{P}$ absent. There are oscillations connecting the ventricular deflections, which are of low voltage $(1 / 2 \mathrm{mV})$. $\mathrm{T}$ round and deformed by oscillations.

Lead III : Action irregular. P indiscernible. Voltage of ventricular complex $1 / 2 \mathrm{mV}$; duration 0.05 sec. $T$ very low in some cycles, wisoelectric in others. There are oscillations between the ventricular complexes.

\section{The Occurrence of Death from Diphtheric Heart-disorders in} Children

Death may occur, sometimes with startling suddenness, in the first ten days of the disease. This often happens in severe cases of the nasopharyngeal type due to the gravis strain of the organism. The fatal issue in these cases is usually brought about by one of three conditions:

A. Gross Myocarditis.-The electrocardiogram shows evidence of extreme myocardial damage.

Case 10: Fig. 16.-Alan W., 9 years : admitted 26/7/1941. Onset 25/7/1941. Severe nasopharyngeal diphtheria K.L.B.+. Antidiphtheria serum 60,000 units intravenously. 28/7/41: Membranes separating. 2/8/41: Vomiting, pallor, cold extremities ; blood pressure 110/84. 3/8/41: Electrocardiogram taken 12 hours before death.

Lead I : Action irregular. R-R 0.85 to $0.87 \mathrm{sec}$. P imperceptible. Ventricular complex splintered; voltage $1 / 5 \mathrm{mV}$; duration 0.16 sec. S-T dislocated. $T$ isoelectric.

Lead II : R-R 0.85 to $0.89 \mathrm{sec}$. P absent. Ventricular complex shows a downward deflection of $1 / 5 \mathrm{mV}$ voltage and $0.15 \mathrm{sec}$ duration. $T$ faintly indicated.

Lead III : R-R 0.80 to $0.87 \mathrm{sec}$. P indiscernible. Ventricular complex: voltage $1 / 3 \mathrm{mV}$, duration 0.1 sec. $T$ isoelectric. 
B. Acute Complete Heart-block (Case 11: Fig. 17).-Rosalie B., 3 years. Onset gradual, about one week. Admitted 11/10/1941. Severe pharyngeal diphtheria. K.L.B.+. Anti-diphtheria serum 30,000 units intraperitoneally. $12 / 10 / 41$ : membranes separating. $16 / 10 / 41$ : blood pressure $76 / 63$ 18/10/41: syncope ; partial a.-v. block ; myocardial lesion. 19/10/41: complete heart-block; pulse 36; cardiac dullness enlarged; liver swollen; vomiting. 20/10/41: pulse rate 20; cyanosis; electrocardingram taken 2 hours before death.

Lead III : Complete a.-v. block. Auricular fibrillation.

C. Ventricular Tachycardia (Case 12: Fig. 18).-Brian D., 3 years, admitted 13/12/1941 ; 4th day of disease. Severe nasopharyngeal diphtheria. 60,000 units anti-diphtheria serum intraperitoneally. Extremely ill; ; pale; hands and feet cold. Heart enlarged ; " ticktack" rhythm. Electrocardiogram shows complete heart-block. 12 p.m.: Child very restless; pulse weak. 14/12/41: semi-conscious ; cyanotic. Offensive oral fetor. Electrocardiogram taken $1 / 2$ hour before death. Regular tachycardia. $R-R \quad 0.49$ sec. Rate 122 . $P$ indiscernible. The ventricular complexes are atypical, and one complex follows the other without any interval. Duration $0.18 \mathrm{sec}$ $\mathrm{T}$ diphasic. There is a longer interval after the last cycle in the third lead.

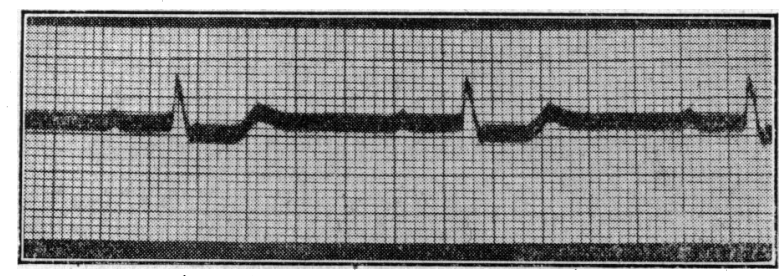

Fig. 9

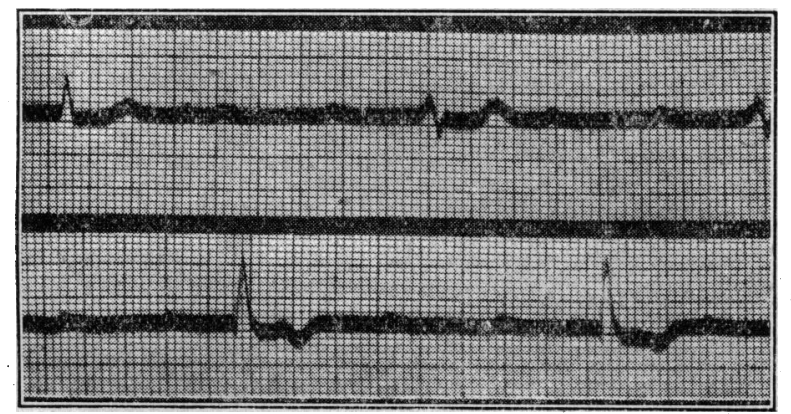

FIG. 10

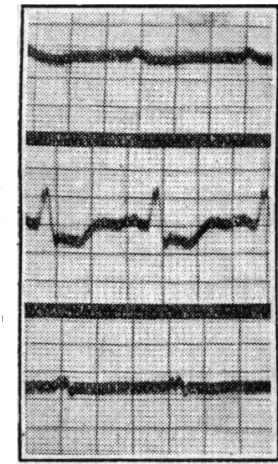

Fig. 11

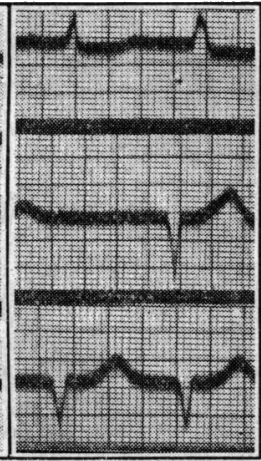

FIG. 12

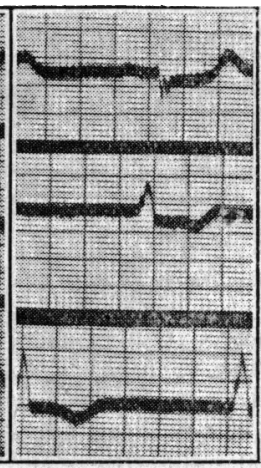

FIG. 13

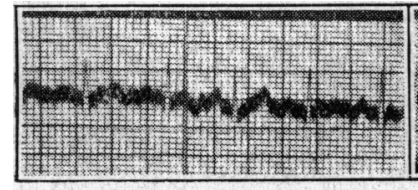

FIG. $14(a) .-+$ Lead II.

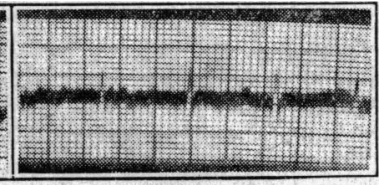

FIG. 14 (b).-Lead III.
The examples given above are sufficient to show that electrocardiographic investigation in support of careful clinical examination of the patient is desirable in the treatment of any case of diphtheria other than the mildest, and especially when there is reason to suspect the presence of myocardial damage.

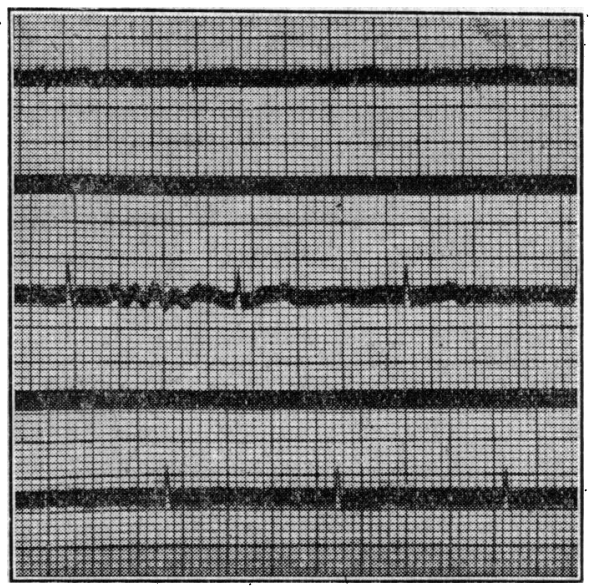

Fig. 15

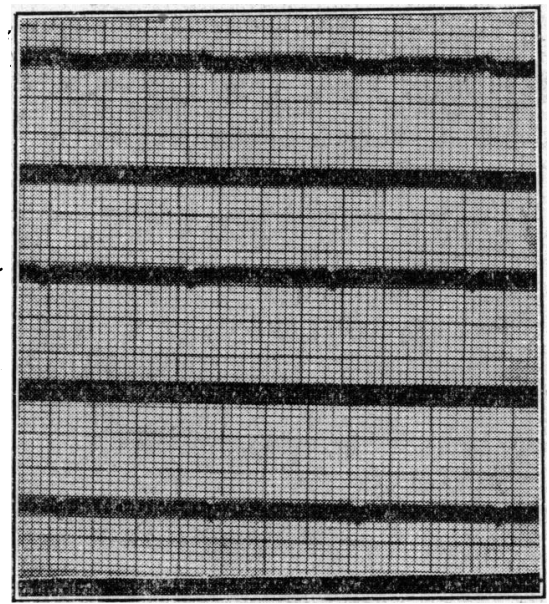

Fig. 16

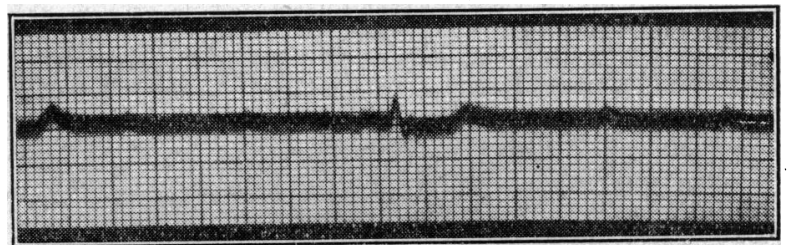

Fio. 17

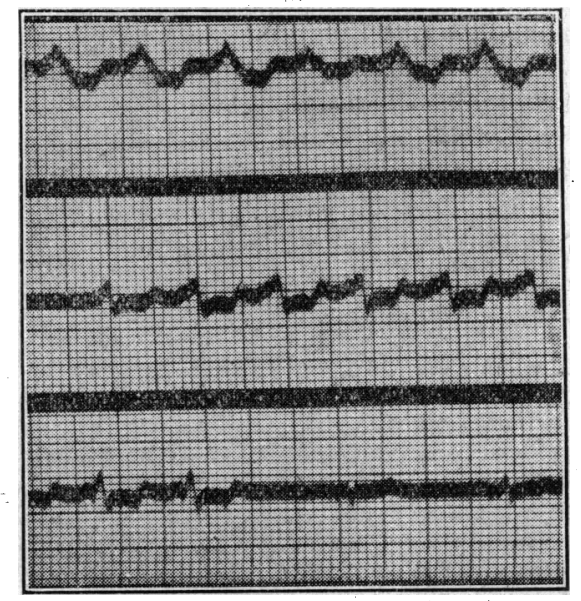

Fic. 18

It is only by the use of the electrocardiogram that a clinical suspicion of myocarditis can be definitely confirmed.

In general it is possible to discharge a patient who has suffered from a mild attack of diphtheria in four weeks, from an attack of moderate severity in six to seven weeks, while those 
with a severe infection may have to be retained in hospital for three months or longer. Where evidence of a myocardial lesion has been found the patient should not be discharged until the electrocardiogram has returned to normal.

I wish to express my thanks to Dr. J. A. Charles, medical officer of health, Newcastle-upon-Tyne, for permission to carry out the investigation, and to Dr. E. F. Dawson-Walker, medical superintendent of the Hospital for Infectious Diseases, Walker Gate, for assistance in the preparation of this article.

$$
\text { Bibliography }
$$

Begg, N. D. (1937). Lancet, 1, 857.

Bower, H. J. (1939), Ibid., 2, 686

Cooper, K. E., Happold, F. C., McLeod, J. W., and Woodcock, H. E. de C. (1936). Proc. roy. Soc. Med., 29, 1029.

Dressler, W. (1932). Klinische Elektrokardiographie, Berlin.

Hoskin, J. (1926). Lancet, 1, 1141

Hume, W. E., and Clegg, S. J. (1914). Quart. J. Med., 8.

Lewis, Sir T. (1937). Clinical Electrocardiography, London.

(1942). Diseases of the Heart, London.

Nathanson, M. H. (1928). Arch. intern. Med., 42, 23.

Shookoff, C., and Taran, L. M. (1931). Amer. J. Dis. Child., 42, 811.

Witt, D. B., Katz, L. N., and Linder, E. (1937). Amer. Heart J., 13, 693.

\section{A CASE OF INTRA-UTERINE INFECTION OF THE FOETUS BY GAS-GANGRENE ORGANISMS}

BY

F. H. KEMP, M.B., M.R.C.P., D.M.R.

Assistant Radiologist to the Radcliffe Infirmary

AND

J. A. STALLWORTHY, F.R.C.S., M.R.C.O.G.

Assistant Obstetrician and Gynaecologist to the Radcliffe Infirmary

The following case is recorded as one of unusual clinical and radiological interest.

\section{Case Report}

The patient, a 2-para aged 30 , was 16 weeks pregnant when she first attended the ante-natal clinic at the Radcliffe Infirmary in November, 1940. Her first child, weighing $6 \mathrm{lb}$. $11 \mathrm{oz}$., was born in 1930, and the second, weighing $9 \mathrm{lb} .8 \mathrm{oz}$., was stillborn the following year. Two months after the second confinement it was discovered that the patient was suffering from diabetes mellitus, and it would seem probable that this condition was present during the pregnancy and was responsible for its unfortunate ending. From the time diabetes was diagnosed in 1931 until the patient attended the Radcliffe Infirmary in 1940 she had required 30 units of insulin daily, and at her first antenatal examination she was found to have just a trace of sugar in the urine, but no acetone or albumin.

Obstetrical progress was uneventful until Feb. 7, 1941, when complaint was made of sickness, and hydramnios was found to be present. The blood pressure was $110 / 80$, and the urine was free of sugar and albumin. Foetal parts could not be felt and, to investigate the cause of the hydramnios, a radiograph was taken. One apparently normal foetus presenting by the vertex was reported. The following week there was a considerable increase in the size of the uterus; acetone and sugar were present in the urine, but there was no albumin. The patient was more uncomfortable and was admitted to hospital. The foetal heart was audible and foetal movements were felt In the next 10 days the abdomen increased rapidly in size and oedema of the lower extremities occurred. At this time the foetal heart could not be heard, so a second radiological examination was made on Feb. 27. It revealed a breech presentation but no overlapping of the skull bones or other features surggesting foetal death. On March 4 the patient stated that she could not feel any foetal movements, and no foetal heart could be heard.

On March 8 a further radiograph revealed a breech presentation with slight overlapping of the skull bones and a large bubble of gas within the shadow of the foetal head (see Fig.). Other films taken with the patient standing upright demonstrated gas and a fluid level within the foetal skull. The foetal brain and its investing membranes seemed to have collapsed, as the air bubble could readily be displaced to different positions by changing the woman's posture. No other bubbles of gas were seen within the foetus. The previous films were reexamined, and the second radiograph, taken on Feb. 27, showed several smaller gas bubbles 'within the shadow of the foetal skull which could not be accounted for by gas in the mother's intestinal canal. The diagnosis was that of intra-uterine foetal death, and it was suggested that there was a gas infection of the foetal skull. Arrangements were made for the pathological laboratories to investigate the foetus bacteriologically as soon as it was delivered.

On March 16 spontaneous delivery of a macerated foetus occurred. The foetal body was soft but the skin was intact. Within an hour of delivery radiographs were taken of the foetal body and the presence of gas in the skull was confirmed. No

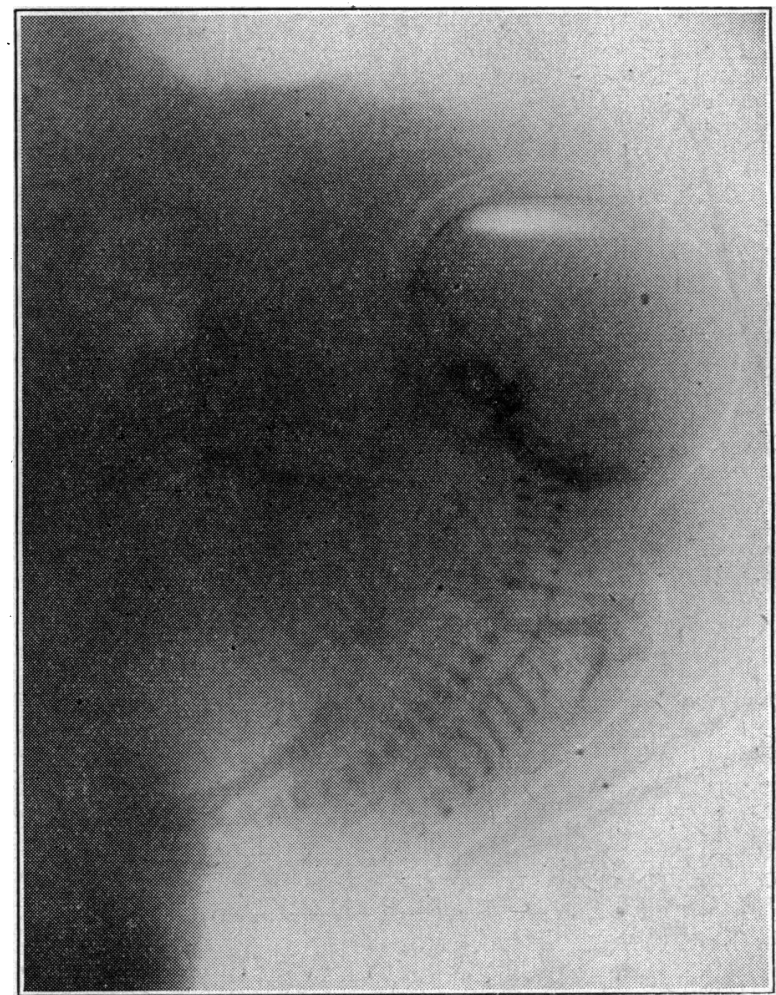

Radiograph taken on March 8. Intracranial gas bubble is well shown.

further collection of gas was seen in any situation. The skeleton was well developed and both lower femoral epiphysial centres were ossified. Dr. A. H. T. Robb-Smith opened the skull, and some of the macerated cranial contents were taken for bacteriological examination. There was extreme maceration but no naked-eye appearance of gas infection of the tissues. Sections from the brain and liver showed an extreme degree of autolysis, but no gas-forming organisms were seen. Bacteriological culture of the smears taken from the cranial contents was reported by Dr. Vollum to show a pure culture of an organism which was identified as $\mathrm{Cl}$. welchii. The puerperium was uneventful, and except for a temperature of $99^{\circ}$ on the third and fourth days it was apyrexial.

It is interesting to note that on Dec. 28, 1941, this patient was delivered of a healthy living female child after spending many weeks in hospital so that her diabetes could be controlled.

\section{Discussion}

This is a case of intra-uterine infection of the foetus with gas-forming organisms occurring in a woman suffering from diabetes mellitus. Hydramnios and increasing oedema of the lower extremities developed at the thirty-second week of gestation, and at this time there was considerable difficulty in controlling diabetes. A fortnight later, at the thirty-fuırth week, there were signs suggesting foetal death, and radiographic examination revealed bubbles of gas within the foetal skull. Seventeen days after this a macerated foetus was delivered and a pure culture of $\mathrm{Cl}$. welchii was obtained from its intracranial contents. There was no evidence of maternal toxaemia due to gas-forming organisms either before or after delivery. It would appear that the foetus died 17 or 18 days before delivery, as the heart had been heard on Feb. 25 but not on the 27th The films taken on the 27 th revealed small collections of gas in the foetal skull. Presumably the infection occurred after the death of the foetus, although it is possible that it was the cause of death. It is difficult to explain the path of 